\title{
The quest for spatio-temporal control of CAR T cells
}

\author{
Cell Research (2015) 25:1281-1282. doi:10.1038/cr.2015.131; published online 17 November 2015
}

Chimeric antigen receptors (CARs) are synthetic receptors capable of directing potent antigen-specific anti-tumor $T$ cell responses. A recent report by $W u$ et al. extends a series of strategies aiming to curb excessive $T$ cell activity, utilizing in this instance a chemical dimerizer to aggregate antigen-binding, $T$ cell-activating and costimulatory domains.

Chimeric antigen receptor (CAR) therapy relies on $\mathrm{T}$ cell engineering to generate tumor-targeted $\mathrm{T}$ cells with enhanced anti-tumor functions [1]. CAR therapy has so far achieved its most remarkable clinical successes against CD19-positive hematological malignancies and is now on the verge of being developed for solid tumors [2]. Two safety concerns have, however, emerged from the CD19 experience, which should be addressed for CAR therapy to be broadly applicable. One is the eventual on-target/off-tumor effect of CAR T cells on normal tissues. Even though this concern may be mitigated in the case of CD19 CAR T cell-induced $\mathrm{B}$ cell aplasia, strategies designed to reduce or prevent its potential occurrence with other targets are needed [2]. The other concern is a severe cytokine release syndrome (CRS), arising from large-scale synchronized $\mathrm{T}$ cell activation upon engaging the target antigen in some CAR T cell recipients [2].

Several innovative strategies have been recently proposed to address these safety concerns. These strategies make use of remote or cell autonomous controls (Figure 1), utilizing small molecules, antibodies or synthetic receptors to regulate $\mathrm{T}$ cell activity. One approach is to activate a latent suicide switch, such as the inducible caspase-9 (iCasp9) enzyme, through the administration of a small molecule to induce $\mathrm{T}$ cell apoptosis [3] (Figure 1a). Bifunctional small molecules that mediate the binding between antigen and CAR have also been developed to regulate target engagement [4] (Figure 1b). A variation on this approach uses antibodies to mediate antigen recognition on target cells and binding of T cells expressing a synthetic Fc receptor [5] (Figure 1b). These designs enable remote temporal control of $T$ cell activity but do not provide a means to enhance tumor selectivity of the CAR T cells. To this end, combinatorial approaches integrating two autonomous antigen inputs to control CAR T cell functions have been developed to spatially discriminate between normal and tumor cells expressing a common target. One such approach utilizes synthetic inhibitory receptors, termed iCARs, which are derived from the PD- 1 or CTLA-4 receptors, to protect normal cells based on the iCAR's recognition of an antigen present on the normal cells but not the tumor cells [6] (Figure 1c). Another approach utilizes complementary signals split between two receptors - a CAR for $\mathrm{T}$ cell activation and a chimeric costimulatory receptor (CCR) providing costimulation - such that they are both expressed by the tumor cells but found alone on normal cells [7] (Figure 1d). Acting in cell autonomous fashion, the required co-engagement of the CCR and the CAR upon recognition of two independent antigens reinforces tumor selectivity in vivo [7].

In a recent paper published in $\mathrm{Sci}$ ence, Wu et al. [8] showed a novel design incorporating a remote control of CAR T cells, whereby a small molecule is used to dimerize antigen-binding and signaling domains (Figure 1e). At variance with the small molecule-controlled suicide switch, this ON-switch design represents a positive reversible regulation, as it does not eliminate $\mathrm{T}$ cells but rather restricts their activities. The remote control takes advantage of well-established chemically induced dimerization (CID) modules developed in the 1990s, where two proteins bind only in the presence of a third chemical, such as a small molecule [9]. One such widely used CID module is the FKBP and $\mathrm{FRB}_{\mathrm{T} 2098 \mathrm{~L}}$ that heterodimerize in the presence of rapamycin or its less immunosuppressive analog AP21976. The receptor for antigen and a dualsignaling, costimulatory and activating domain analogous to that of a second generation CAR, were independently fused to FKBP and $\mathrm{FRB}_{\mathrm{T} 2098 \mathrm{~L}}$ so that AP21976-induced FKBP and FRB ${ }_{\text {T2098L }}$ dimerization could aggregate these entities (Figure 1e). This design controls intracellular assembly of a signaling complex without affecting the antigen binding properties as afforded by the bifunctional small molecules or antibodies at the interface of $\mathrm{T}$ cells and target cells (Figure 1b). After screening various domain configurations in leukemic Jurkat cells with AP21976-dependent NFAT activation and IL-2 production assays, a design that worked with both the FKBP-FRB ${ }_{\text {T2098 }}$ and the gibberellininduced GID1-GAI heterodimerization modules was identified. Single molecule imaging of ON-switch CAR assembly in Jurkat cells showed that two molecular parts are equally constrained to im- 


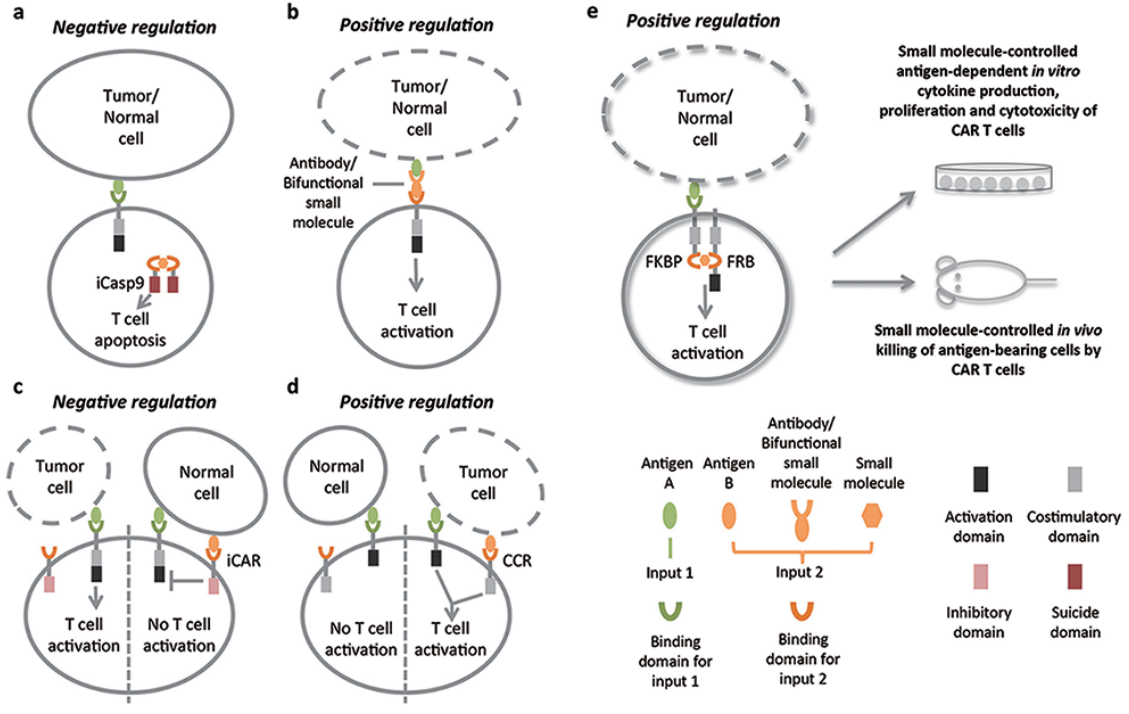

Figure 1 Building controls into engineered T cells. (a) The small molecule AP1903 can dimerize the suicide switch iCasp9 to induce T cell apoptosis. (b) Bifunctional small molecule bridging the binding between antigen and CAR or antibody mediating the interaction between antigen and synthetic Fc receptor can be remote controls of CAR T cells. (c) iCAR can inhibit CAR function in the presence of an antigen present in normal cells but not tumor cells. (d) CCR binding to a second antigen in tumor cells is required for full T cell activation. (e) The small molecule AP21976 can dimerize two independent signaling entities through an FKBP-FRB module to control $T$ cell activation. (a, b, e) Strategies employing one remote control (antibody or small molecule) in addition to one autonomous control (antigen A). (c, d) Strategies with two autonomous controls (antigen $\mathrm{A}$ and antigen $\mathrm{B}$ ). Negative regulation involves inducing apoptosis (a) or turning off T cell activation (c) by input 2 while positive regulation $(\mathbf{b}, \mathbf{d}, \mathbf{e})$ results in T cell activation by input 2 .

mobilized antigens only in the presence of AP21976. Subsequent characterization of the ON-switch CAR in primary human $\mathrm{CD}^{+} \mathrm{T}$ cells showed that both AP21976 and antigen are required for the induction of CD69 expression, a biomarker of $\mathrm{T}$ cell activation, the secretion of both IL-2 and IFN $\gamma$, and the proliferation of $\mathrm{CD}^{+}$cells. Most gratifyingly, there was a positive correlation between these responses and the AP21976 dosage, suggesting the possibility of achieving titratable control of $\mathrm{T}$ cells. Human primary $\mathrm{CD} 8^{+} \mathrm{T}$ cells with ON-switch CAR in three different cytotoxicity assays also demonstrated antigen- and AP21976-dependent killing of tumor cells, which was also titratable by AP21976. The killing ability of ON-switch CAR $\mathrm{CD}^{+} \mathrm{T}$ cells was reversible, as removal of AP2 1976 abrogated tumor cell lysis.
Wu et al. proceeded to explore in vivo activity in a mouse xenograft model. Due to the short plasma half-life and the high cost of AP21976, the study is limited to a very short-term protocol of $39 \mathrm{~h}$. Tumor cells were injected into the peritoneal cavity $14 \mathrm{~h}$ prior to the injection of the engineered T cells. Four injections of AP21976 in the subsequent $25 \mathrm{~h}$ were required to induce anti-tumor activity in this intraperitoneal cytotoxicity assay. Further investigations with a more relevant protocol allowing for tumor engraftment and longer term follow-up of T cell effectiveness will be needed to establish whether AP21976 can remotely control ON-switch CAR $\mathrm{T}$ cells to reject a tumor.

$\mathrm{Wu}$ and coauthors have thus engineered a novel ON-switch CAR design and demonstrated titratable, reversible and antigen-dependent $\mathrm{T}$ cell functions controlled by a dimerizing small molecule. Another group is also conducting preclinical studies exploring a variant small molecule-controlled CAR design for solid tumor rejection [10]. However, there are still challenges to address before future clinical applications. The authors pointed out the need to develop controller chemicals that have clinically optimized pharmacokinetic properties, as the half-life of AP21976 is short and impractical for clinical application. Thus, how many injections per day, for how many weeks or months, would be required to achieve tumor rejection? Another unresolved question is whether a small molecule with optimal pharmacokinetic properties could effectively curb CRS and off-tumor reactivity. Overall, this elegant study provides valuable insights for further refining spatio-temporal control of cell therapy and applying it to CAR T cell technology.

\section{Jie Sun ${ }^{1,2}$, Michel Sadelain ${ }^{1,2}$}

${ }^{1}$ Center for Cell Engineering, Memorial Sloan Kettering Cancer Center, New York, NY 10065 , USA; '2Immunology Program, Sloan Kettering Institute, New York, NY 10065, USA Correspondence: Michel Sadelain E-mail: m-sadelain@ski.mskcc.org

\section{References}

1 Jensen MC, Riddell SR. Curr Opin Immunol 2015; 33:9-15.

2 Sadelain M. J Clin Invest 2015; 125:33923400 .

3 Di Stasi A, Tey SK, Dotti G, et al. N Engl J Med 2011; 365:1673-1683.

4 Kim MS, Ma JS, Yun H, et al. J Am Chem Soc 2015; 137:2832-2835.

5 Kudo K, Imai C, Lorenzini P, et al. Cancer Res 2014; 74:93-103.

6 Fedorov VD, Themeli M, Sadelain M. Sci Transl Med 2013; 5:215ra172.

7 Kloss CC, Condomines M, Cartellieri M, et al. Nat Biotechnol 2013; 31:71-75.

8 Wu CY, Roybal KT, Puchner EM, et al. Science 2015; 350:aab4077.

9 Spencer DM, Wandless TJ, Schreiber SL, et al. Science 1993; 262:1019-1024.

10 http://www.bellicum.com/technology/gocart/ 